MIT-CTP-3008

HLRZ2000_13

\title{
Approximate Ginsparg-Wilson fermions: A first test
}

\author{
Christof Gattringer ${ }^{a \dagger}$, Ivan Hip ${ }^{b}$ and C. B. Lang ${ }^{c}$ \\ ${ }^{a}$ Massachusetts Institute of Technology, Center for Theoretical Physics \\ 77 Massachusetts Avenue, Cambridge MA 02139 USA \\ ${ }^{b}$ NIC - John von Neumann Institute for Computing \\ FZ-Jülich, 52425 Jülich, Germany \\ ${ }^{c}$ Institut für theoretische Physik, Karl-Franzens Universität Graz \\ Universitätsplatz 5, A-8010 Graz, Austria
}

\begin{abstract}
We construct a 4-d lattice Dirac operator $D$ using a systematical expansion in terms of simple operators on the lattice. The GinspargWilson equation turns into a system of coupled equations for the expansion coefficients of $D$. We solve these equations for a finite parametrization of $D$ and find an approximate solution of the Ginsparg-Wilson equation. We analyze the spectral properties of our $D$ for various ensembles of quenched SU(3) configurations. Improving the gauge field action considerably improves the spectral properties of our $D$.
\end{abstract}

PACS: 11.15.Ha

Key words: Lattice field theory, chiral fermions

† Supported by the Austrian Academy of Sciences (APART 654). 


\section{Introduction}

Chiral symmetry in fermionic field theories on the lattice may be implemented by adding a non-vanishing and in the continuum limit irrelevant right hand side of the usual anti-commutator of the Dirac operator $D$ with $\gamma_{5}$. In its simplest form the resulting so-called Ginsparg-Wilson equation [1] reads (we set the lattice spacing to 1 )

$$
D \gamma_{5}+\gamma_{5} D=D \gamma_{5} D \text {. }
$$

Based on (1.1) chirally symmetric fermions can be constructed on the lattice (for reviews of recent developments see e.g. [2, 3, 4]).

Currently two types of solutions for (1.1) are known. Neuberger [5] gave an explicit construction, the so-called overlap operator based on earlier work, the overlap approach to chiral fermions on the lattice [6]. The overlap operator is given by

$$
D=1-A\left(A^{\dagger} A\right)^{-\frac{1}{2}} \quad \text { with } \quad A=1+s-D_{0} .
$$

Here $D_{0}$ is some decent lattice Dirac operator which is free of doublers. Typically the Wilson-Dirac operator is used here but also other choices give rise to solutions of (1.1); $s$ is a real number which can, at the cost of an additional renormalization, be used to optimize the locality properties of $D$ [7]. The main challenge when using the overlap operator is the computation of the square root in (1.2), making simulations with the overlap operator considerably more expensive than simulations with the Wilson operator.

The second solution to (1.1) is provided by the fixed point Dirac operator [8, 9] which goes back to the perfect action approach to lattice field theories [10]. So far, the fixed point Dirac operator has been computed only in two dimensions 111, 12, 13] and it has become clear that its construction in 4-d is quite a challenging enterprise.

Recently [14] a new line of attack for solving the Ginsparg-Wilson equation has been proposed. The basic idea is to systematically expand the most general lattice Dirac operator $D$ in a series of simple basis operators on the lattice. This expanded $D$ is then inserted into the Ginsparg-Wilson equation. The product $D \gamma_{5} D$ on the right hand side of (1.1) can be evaluated using techniques from the analytical hopping expansion. The result is an expansion of both sides of (1.1); comparing the factors in front of the contributions to this expansion one finds that the Ginsparg-Wilson equation is mapped to an equivalent system of coupled quadratic equations for 
the expansion coefficients of $D$. When using finitely many terms in the expansion of $D$, the system of equations for the expansion coefficients can be solved numerically and the corresponding $D$ is an approximate solution of the Ginsparg-Wilson equation. By including more terms in the expansion of $D$ the violation of (1.1) can be made arbitrarily small.

Here we present first results in 4-d for the discussed method. We explicitly construct an approximate solution $D$ of (1.1) and study its properties in different ensembles of quenched $\mathrm{SU}(3)$ gauge configurations. We find that already with relatively few terms we obtain a good approximate solution for the Ginsparg-Wilson equation. We discuss properties of the spectrum of our Dirac operator and also analyze the role of improving the gauge field action. We demonstrate that improving the gauge field action considerably improves the spectral properties of our $D$.

We would like to point out three aspects of our approximate solutions of the Ginsparg-Wilson equation:

- In an earlier study 15 in two dimensions we had constructed an approximate solution of (1.1) using the outlined ideas. It turned out that essential features of chiral symmetry in the lattice Schwinger model were properly described by our approximate solution of the GinspargWilson equation. It will be interesting to see if our $D$ is able to capture chiral symmetry also in four dimensions.

- Our study should help to shed light on the importance of different terms in solutions $D$ of the Ginsparg-Wilson equation. In particular for the perfect action program an economical but still rich enough parametrization of $D$ is essential [16].

- Another application of our $D$ is its use as a starting operator $D_{0}$ in Neuberger's construction. Using an approximate solution of (1.1) in the projection (1.2) has been proposed previously [17] and it is believed that an improved $D_{0}$ speeds up the evaluation of the square root and helps to overcome the problems with the potential singularity of (1.2) for small eigenvalues of $A$.

The article is organized as follows: In Section 2 we briefly discuss the expansion of $D$ and derive the system of equations equivalent to the GinspargWilson equation. We discuss in Section 3 the boundary conditions and find a solution of the system of coupled equations. In Section 4 we analyze the spectral properties of our approximate solution D for ensembles of quenched gauge field configurations. We start with the free case in Section 4.1 followed 
by gauge field ensembles generated with the standard Wilson gauge action (Section 4.2). Finally in Section 4.3 we investigate how improving the gauge field action can further improve the chiral properties of the lattice Dirac operator. The article closes with a discussion in Section 5. In particular we will come back to the above mentioned three possible applications of our Dirac operator.

\section{Constructing approximate solutions of the Ginsparg-Wilson equation}

To make the article self contained we briefly repeat the basic idea of our construction presented in [14]. In a first step we discuss the systematic expansion of the most general Euclidean Dirac operator in a series of simple operators on the lattice. Subsequently we derive the system of quadratic equations for the expansion coefficients which is equivalent to the GinspargWilson equation.

\subsection{Expansion of the most general $D$}

Typically the derivative term on the lattice is discretized by the following nearest neighbor term (we set the lattice spacing to 1 ):

$$
\frac{1}{2} \sum_{\mu=1}^{4} \gamma_{\mu}\left[U_{\mu}(x) \delta_{x+\hat{\mu}, y}-U_{\mu}(x-\hat{\mu})^{-1} \delta_{x-\hat{\mu}, y}\right] .
$$

However, it is perfectly compatible with all the symmetries to instead discretize the derivative term using

$$
\frac{1}{4} \sum_{\mu=1}^{4} \gamma_{\mu}\left[U_{\mu}(x) U_{\mu}(x+\hat{\mu}) \delta_{x+2 \hat{\mu}, y}-U_{\mu}(x-\hat{\mu})^{-1} U_{\mu}(x-2 \hat{\mu})^{-1} \delta_{x-2 \hat{\mu}, y}\right],
$$

and there are many more terms one could think of. Thus an ansatz for the most general Dirac operator $D$ must allow for a superposition of all of the possible discretizations for the derivative term.

The terms in (2.1) and (2.2) can be characterized by simple paths on the lattice. The first example (2.1) consists of a single hop in positive $\mu$-direction with a plus sign and a single hop in negative direction with a minus sign. Similarly the second example consists of two hops in positive (negative) $\mu$ direction. We now introduce a shorthand notation for such paths using an 
ordered list of the directions of the links of the path. We denote a path of length $n$ on the lattice by

$$
<l_{1}, l_{2}, \ldots l_{n}>
$$

with the $l_{i}$ giving the directions of the subsequent hops, i.e. $l_{i} \in\{ \pm 1, \pm 2, \pm 3$, $\pm 4\}$. It is implicitly understood, that each link is dressed with the corresponding link variable $U_{\mu}(x)$. Using this notation the terms from the two examples (2.1) and (2.2) are denoted as

$$
\frac{1}{2} \sum_{\mu} \gamma_{\mu} \sum_{l= \pm \mu} s(l)<l>
$$

and

$$
\frac{1}{4} \sum_{\mu} \gamma_{\mu} \sum_{l= \pm \mu} s(l)<l, l>
$$

We use the abbreviation $s(l)$ for $\operatorname{sign}(l)$. Due to translation invariance the form of the derivative terms, and thus of the paths, is the same at all lattice points such that in our notation no reference to the starting point for the paths is necessary.

In order to remove the doublers one also needs a term which in momentum space can distinguish between $p_{\mu}=0$ (physical modes) and $p_{\mu}=\pi$ (doublers). Such a term is provided by the standard Wilson term. Due to the symmetries this term has to come with $\mathbb{I}$ in spinor space. Again we will allow for all possible terms. We generalize our $D$ further, by including all terms also for the remaining elements $\Gamma_{\alpha}$ of the Clifford algebra, i.e. tensors, pseudovectors and the pseudoscalar. Thus the emerging lattice Dirac operator has the following form:

$$
D=\sum_{\alpha=1}^{16} \Gamma_{\alpha} \sum_{p \in \mathcal{P}^{\alpha}} c_{p}^{\alpha}<l_{1}, l_{2}, \ldots l_{|p|}>.
$$

To each generator $\Gamma_{\alpha}$ of the Clifford algebra we assign a set $\mathcal{P}^{\alpha}$ of paths $p$, each $p$ given by some ordered set of links $\left\langle l_{1}, l_{2}, \ldots l_{|p|}>\right.$ (compare (2.3)) where $|p|$ denotes the length of the path $p$. Each path is weighted with some complex weight $c_{p}^{\alpha}$.

The next step is to impose on $D$ the symmetries which we want to maintain: Translation and rotation invariance and invariance under $\mathrm{C}$ and $\mathrm{P}$. In addition we require our $D$ to be $\gamma_{5}$-hermitian, i.e. we require $\gamma_{5} D \gamma_{5}=D^{\dagger}$. This property can be seen to correspond to what leads to the CPT theorem 
in Minkowski space, i.e. the vector generators $\gamma_{\mu}$ come with a derivative term etc.

Translation invariance has already been briefly mentioned above and requires the sets $\mathcal{P}^{\alpha}$ of paths and their coefficients to be independent of the starting point. Rotation invariance implies that a path and its rotated image have the same weight. Parity implies that for each path $p$ (with coefficient $c_{p}^{\alpha}$ ) we must include the parity-reflected copy with coefficient $s_{\text {parity }}^{\alpha} \cdot c_{p}^{\alpha}$ where the signs $s_{\text {parity }}^{\alpha}$ are defined by $\gamma_{4} \Gamma_{\alpha} \gamma_{4}=s_{\text {parity }}^{\alpha} \cdot \Gamma_{\alpha}$.

Of importance are the symmetries $\mathrm{C}$ and $\gamma_{5}$-hermiticity. It is easy to see that both of them imply a relation between the coefficient for a path $p$ and the coefficient of the inverse path $p^{-1}$. Implementing both these symmetries restricts all coefficients $c_{p}^{\alpha}$ to be either real or purely imaginary. Furthermore we find that the coefficient for a path $p$ and the coefficient for its inverse $p^{-1}$ are equal up to a sign $s_{\text {charge }}^{\alpha}$ defined by $C \Gamma_{\alpha} C=s_{\text {charge }}^{\alpha} \cdot \Gamma_{\alpha}^{T}$, where $T$ denotes transposition and $C$ is the charge conjugation matrix.

When implementing all these symmetries we find that paths in our ansatz become grouped together where - up to sign factors - all paths in a group come with the same coefficient. We can now write down our most general Dirac operator on the lattice in the form (compare [14 and see also [16 for an equivalent derivation using a slightly different notation):

$$
\begin{aligned}
& D \equiv \mathbb{I}\left[s_{1}<>+s_{2} \sum_{l_{1}}<l_{1}>+s_{3} \sum_{l_{2} \neq l_{1}}<l_{1}, l_{2}>+s_{4} \sum_{l_{1}}<l_{1}, l_{1}>\ldots\right] \\
& +\sum_{\mu} \gamma_{\mu} \sum_{l_{1}= \pm \mu} s\left(l_{1}\right)\left[v_{1}<l_{1}>+v_{2} \sum_{l_{2} \neq \pm \mu}\left[<l_{1}, l_{2}>+<l_{2}, l_{1}>\right]\right. \\
& +\sum_{\mu<\nu} \gamma_{\mu} \gamma_{\nu} \sum_{\substack{l_{1}= \pm \mu \\
l_{2}= \pm \nu}} s\left(l_{1}\right) s\left(l_{2}\right) \sum_{i, j=1}^{2} \epsilon_{i j}\left[t_{1}<l_{i}, l_{j}>\ldots\right] \\
& +\sum_{\mu<\nu<\rho} \gamma_{\mu} \gamma_{\nu} \gamma_{\rho} \sum_{l_{1}= \pm \mu, l_{2}= \pm \nu} s\left(l_{1}\right) s\left(l_{2}\right) s\left(l_{3}\right) \sum_{i, j, k=1}^{3} \epsilon_{i j k}\left[a_{1}<l_{i}, l_{j}, l_{k}>\ldots\right] \\
& +\sum_{l_{3}= \pm \rho} s\left(l_{1}\right) s\left(l_{2}\right) s\left(l_{3}\right) s\left(l_{4}\right) \sum_{i, j, k, n=1}^{4} \epsilon_{i j k n}\left[p_{1}<l_{i}, l_{j}, l_{k}, l_{n}>\ldots\right] .
\end{aligned}
$$

By $\epsilon$ we denote the totally anti-symmetric tensors with 2,3 and 4 indices. We choose the normalization of the elements of the Clifford algebra such that 
the elements appear as all possible products of the $\gamma_{\mu}$ without any extra factors of $i$. For this normalization the symmetries $\mathrm{C}$ and $\gamma_{5}$-hermiticity render all coefficients $s_{i}, v_{i}, t_{i}, a_{i}$ and $p_{i}$ real. To be specific, we use the Euclidean chiral representation for the $\gamma_{\mu}=\gamma_{\mu}^{\dagger}$. In principle it would be possible to generalize $D$ further by multiplying each term with a polynomial of traces of gauge field variables around closed loops on the lattice, but we do not include this possibility here.

The above mentioned structure of paths appearing in groups is obvious. The paths in each group are related by symmetries and up to the sign factors have to come with the same real coefficient. All paths within a group have the same length.

It has to be stressed, that in (2.7) for each generator we show only the leading terms of an infinite series of groups of paths. The dots indicate that we omitted groups with paths that are longer than the terms we display. It is known [18 that no ultra-local solutions of the Ginsparg-Wilson equation exist and thus an expansion for a solution of (1.1) necessarily contains infinitely many terms. Eventually we will work with a finite $D$ (the corresponding truncation will be discussed in Section 3) but for the moment we keep deriving the method in its most general form, i.e. containing no truncation.

\subsection{The system of coupled equations corresponding to the Ginsparg-Wilson equation}

Let us now insert our expanded Dirac operator $D$ into the Ginsparg-Wilson equation. To that purpose we multiply (1.1) with $\gamma_{5}$ from the left, bring the terms linear in $D$ to the right-hand side and define:

$$
E \equiv-D-\gamma_{5} D \gamma_{5}+\gamma_{5} D \gamma_{5} D
$$

We remark that $E$ is hermitian since we implemented $\gamma_{5}$-hermiticity for $D$. Finding a solution $D$ of the Ginsparg-Wilson equation corresponds to having $E=0$. Evaluating the linear part of $E$ is straightforward: When evaluating the product $\gamma_{5} D \gamma_{5}$ we find that the terms with an odd number of $\gamma_{\mu}$, i.e. vector- and pseudovector terms pick up a minus sign, while the other terms remain unchanged. Thus when adding the two linear terms we find that the terms with an odd number of $\gamma_{\mu}$ cancel, while the other terms pick up a factor of 2 .

The next step is to compute the quadratic term $\gamma_{5} D \gamma_{5} D$. Here we have to multiply the various terms appearing in $D$. Each term is made out of two parts, a generator of the Clifford algebra and a group of paths. The 
multiplication of two of these terms proceeds in two steps: First the two elements of the Clifford algebra are multiplied giving again an element of the algebra. In the second step we have to multiply the paths of our two terms. This multiplication can be noted very conveniently in our notation, where multiplication of two paths simply consists of writing the paths into one long path:

$$
<l_{1}, l_{2} \ldots l_{n}>\times<l_{1}^{\prime}, l_{2}^{\prime} \ldots l_{n^{\prime}}^{\prime}>=<l_{1}, l_{2} \ldots l_{n}, l_{1}^{\prime}, l_{2}^{\prime} \ldots l_{n^{\prime}}^{\prime}>.
$$

It is straightforward to establish this rule by translating back to the algebraic expression of our examples (2.1), (2.2) and performing the multiplication in this notation. It can happen that after multiplying two paths a hop in some direction $l_{i}$ is immediately followed by its inverse $-l_{i}$. These two hops then cancel each other and we find

$$
<l_{1} \ldots l_{i-1}, l_{i},-l_{i}, l_{i+1} \ldots l_{n}>=<l_{1} \ldots l_{i-1}, l_{i+1} \ldots l_{n}>.
$$

This rule is used to reduce all products of paths appearing in $\gamma_{5} D \gamma_{5} D$ to their true length. In a final step we decompose the product terms into groups related by the symmetries in the same way as we did above when constructing the most general ansatz for $D$. Adding the linear and quadratic terms of (2.8) we end up with the following expansion for $E$ :

$$
\begin{aligned}
& E \equiv \mathbb{I}\left[e_{1}^{s}<>+e_{2}^{s} \sum_{l_{1}}<l_{1}>+e_{3}^{s} \sum_{l_{2} \neq l_{1}}<l_{1}, l_{2}>+e_{4}^{s} \sum_{l_{1}}<l_{1}, l_{1}>\ldots\right] \\
& +\sum_{\mu} \gamma_{\mu} \sum_{l_{1}= \pm \mu} s\left(l_{1}\right)\left[e_{1}^{v} \sum_{l_{2} \neq \pm \mu}\left[<l_{1}, l_{2}>-<l_{2}, l_{1}>\right] \ldots\right] \\
& +\sum_{\mu<\nu} \gamma_{\mu} \gamma_{\nu} \sum_{\substack{l_{1}= \pm \mu \\
l_{2}= \pm \nu}} s\left(l_{1}\right) s\left(l_{2}\right) \sum_{i, j=1}^{2} \epsilon_{i j}\left[e_{1}^{t}<l_{i}, l_{j}>\ldots\right] \\
& +\sum_{\mu<\nu<\rho} \gamma_{\mu} \gamma_{\nu} \gamma_{\rho} \sum_{l_{1}= \pm \mu, l_{2}= \pm \nu} s\left(l_{1}\right) s\left(l_{2}\right) s\left(l_{3}\right) \sum_{i, j, k=1}^{3} \epsilon_{i j k}\left[\sum _ { l _ { 4 } \neq \pm \mu , \nu , \rho } \left\{e _ { 1 } ^ { a } \left[<l_{i}, l_{j}, l_{k}, l_{4}>\right.\right.\right. \\
& \left.\left.\left.-\quad<l_{4}, l_{i}, l_{j}, l_{k}>\right]+e_{2}^{a}\left[<l_{i}, l_{4}, l_{j}, l_{k}>-<l_{i}, l_{j}, l_{4}, l_{k}>\right]\right\} \ldots\right] \\
& +\gamma_{5} \sum_{\substack{l_{1}= \pm 1, l_{2}= \pm 2 \\
l_{3}= \pm 3, l_{4}= \pm 4}} s\left(l_{1}\right) s\left(l_{2}\right) s\left(l_{3}\right) s\left(l_{4}\right) \sum_{i, j, k, n=1}^{4} \epsilon_{i j k n}\left[e_{1}^{p}<l_{i}, l_{j}, l_{k}, l_{n}>\ldots\right] .
\end{aligned}
$$

We remark that all the algebraic steps leading to the expansion of $E$ are straightforward to formalize and for the higher orders in the expansion we 
used a computer program. As for $D$, the expansion of $E$ is an infinite series and we display here only the leading groups of paths. The coefficients $e_{i}^{\alpha}$ are now quadratic polynomials in the original coefficients $s_{i}, v_{i}, t_{i}, a_{1}$ and $p_{i}$ given by

$$
\begin{aligned}
e_{1}^{s}= & -2 s_{1}+s_{1}^{2}+8 s_{2}^{2}+48 s_{3}^{2}+8 s_{4}^{2}+8 v_{1}^{2}+96 v_{2}^{2}+8 v_{3}^{2}+48 t_{1}^{2}+192 a_{1}^{2} \\
& +384 p_{1}^{2} \ldots, \\
e_{2}^{s}= & -2 s_{2}+2 s_{1} s_{2}+12 s_{2} s_{3}+2 s_{2} s_{4}+12 v_{1} v_{2}+2 v_{1} v_{3} \ldots, \\
e_{3}^{s}= & -2 s_{3}+2 s_{1} s_{3}+s_{2}^{2}+4 s_{3}^{2}+2 s_{3} s_{4}+4 v_{2}^{2}+2 v_{2} v_{3} \ldots, \\
e_{4}^{s}= & -2 s_{4}+2 s_{1} s_{4}+s_{2}^{2}+6 s_{3}^{2}-v_{1}^{2}-6 t_{1}^{2}-24 a_{1}^{2}-48 p_{1}^{2} \ldots, \\
e_{1}^{v}= & -s_{2} v_{1}-4 s_{3} v_{2}-2 s_{4} v_{2}-s_{3} v_{3}-v_{3} t_{1}-4 v_{2} t_{1} \ldots, \\
e_{1}^{t}= & -2 t_{1}+2 s_{1} t_{1}-2 s_{4} t_{1}-v_{1}^{2}-4 v_{2}^{2}-2 v_{2} v_{3}-4 t_{1}^{2}+8 v_{1} a_{1}-8 a_{1}^{2} \\
& +16 t_{1} p_{1} \ldots \\
e_{1}^{a}= & -s_{2} a_{1}+v_{2} t_{1}-v_{3} p_{1} \ldots, \\
e_{2}^{a}= & -v_{2} t_{1} \ldots, \\
e_{3}^{a}= & -s_{2} a_{1}-2 v_{2} p_{1} \ldots, \\
e_{4}^{a}= & -2 s_{2} a_{1}+2 v_{2} t_{1}-4 v_{2} p_{1} \ldots, \\
e_{1}^{p}= & -2 p_{1}+2 s_{1} p_{1}-2 s_{4} p_{1}-2 v_{1} a_{1}+t_{1}^{2} \ldots
\end{aligned}
$$

Due to different symmetry properties ( $E$ is hermitian, $D$ is $\gamma_{5}$-hermitian) there are terms in $E$ which do not occur in $D$ and vice versa. Each coefficient itself is an infinite series of terms. For a solution of the Ginsparg-Wilson equation we must have $E=0$. It is easy to see, that the groups of paths appearing in the expansion (2.11) are linearly independent and hence for $E=0$ all coefficients $e_{i}^{\alpha}$ have to vanish simultaneously. Thus we have rewritten the problem of finding a solution of the Ginsparg-Wilson equation to solving the system (2.12) of coupled quadratic equations (set all righthand sides $\left.e_{i}^{\alpha}=0\right)$.

\section{Solving the system of coupled equations}

In the last section we have shown that the Ginsparg-Wilson equation is equivalent to a system of coupled quadratic equations for the expansion coefficients of $D$. In this section we now truncate our expansion of $D$ and find solutions for the coupled equations. Before we do this let us discuss the boundary conditions which have to be added to the system (2.12). 


\subsection{Boundary conditions}

For the free case the situation is simple: In this case we can compute the Fourier transform $\hat{D}(p)$ of $D$. For small momenta the massless Dirac operator should obey

$$
\hat{D}(p) \equiv i \not p+\mathcal{O}\left(p^{2}\right) .
$$

This leads to two more supplementary equations for the coefficients, one for the constant term to vanish and the second one sets the slope of the dispersion relation equal to one:

$$
\begin{aligned}
& 0=s_{1}+8 s_{2}+48 s_{3}+8 s_{4} \ldots \\
& 1=2 v_{1}+24 v_{2}+4 v_{3} \ldots
\end{aligned}
$$

We remark, that (3.2) is implicitly guaranteed by the Ginsparg-Wilson equation, since the Ginsparg-Wilson circle円 runs through the origin. When we construct approximate solutions below it will be necessary to explicitly implement (3.2). When we started to solve the system of equations (2.12) respecting the boundary conditions (3.2), (3.3) we found, that for this setting one has to include a relatively large number of terms in the expansion (2.7) of $D$. Since every new term in $D$ drives up the cost of a numerical treatment of our Dirac operator we decided to allow for an additional freedom in our equations. The idea is to allow some $\beta$-dependence by introducing a new parameter, similar in spirit to the variable $s$ in the overlap construction (1.2), which may be used to improve locality of $D$, i.e. to make higher terms in the expansion (2.7) of $D$ less important. At the same time we do not want to change our coupled equations (2.12) since they guarantee that - up to a certain order - we deal with a solution of the Ginsparg-Wilson equation. Thus we only modify the boundary conditions (3.2), (3.3).

Before we discuss the modifications let us first develop the idea for the case of the standard Wilson action. Wilson's lattice Dirac operator $D_{W}$ is a sum of a constant and a hopping term $H$

$$
\begin{aligned}
& D_{W}=4-\kappa H \\
& H_{x, y}=\sum_{\mu}\left\{\left[1-\gamma_{\mu}\right] U_{\mu}(x) \delta_{x+\mu, y}+\left[1+\gamma_{\mu}\right] U_{\mu}(x-\mu)^{-1} \delta_{x-\mu, y}\right\} .
\end{aligned}
$$

\footnotetext{
${ }^{1}$ It is well known, that an exact, $\gamma_{5}$-hermitian solution of the Ginsparg-Wilson equation has its spectrum on the so-called Ginsparg-Wilson circle, i.e. the circle of radius 1 with center 1 in the complex plane.
} 
For the free case the Fourier transform of the hopping matrix is given by $\hat{H}(p)=8-i 2 \not p+\mathcal{O}\left(p^{2}\right)$. Thus the boundary condition corresponding to (3.1) reads

$$
4-8 \kappa+2 i \kappa \not p+\mathcal{O}\left(p^{2}\right) \equiv i \not p+\mathcal{O}\left(p^{2}\right),
$$

and from this equation one finds that for the free case the correct value of the parameter is given by $\kappa=1 / 2$. In fact, in the notation of our parametrization of $D$ we have $s_{1}=4, s_{2}=-\kappa$ and $v_{1}=\kappa$ with all other coefficients vanishing. The condition (3.5) for the Wilson operator therefore corresponds to both our boundary conditions (3.2) and (3.3). In principle one could generalize the Wilson action and lift the degeneracy between $-s_{2}$ and $v_{1}$ which would amount to two boundary conditions similar to (3.2), (3.3) instead of the single condition (3.5).

The situation changes when we include gauge fields. One finds that in order to drive the system critical one needs to change the value of $\kappa$. E.g. when analyzing quenched $\mathrm{SU}(3)$ gauge theory at $\beta=6.0$ one finds $\kappa=0.624$ (see below). Obviously this value of kappa is not a solution of the boundary condition (3.5) but instead solves the more general equation

$$
4-8 \kappa z+2 i \kappa z \not p+\mathcal{O}\left(p^{2}\right) \equiv i \not p+\mathcal{O}\left(p^{2}\right) .
$$

Here $z$ is a real function of $\beta$. For quenched gauge fields at $\beta=6$, we find e.g. $z=1.603=1 / 0.624$.

We now generalize the boundary conditions (3.2), (3.3) analogous to (3.6) being a generalization of (3.5). In the boundary condition we multiply each coefficient by a power $z^{n}$ of some real, $\beta$-dependent number $z$. The exponent $n$ is given by the number of hops of the corresponding term in our expansion (2.7). Thus e.g. the coefficient $s_{1}$, which is the constant term, does not get changed at all, $s_{2}$ which is the coefficient for the single hop obtains a factor of $z, s_{3}$ which corresponds to two hops becomes multiplied by $z^{2}$ et cetera. We allow for two different coefficients $z_{s}$ and $z_{v}$ in the scalar and vector sectors. Our generalized boundary conditions then read

$$
\begin{aligned}
0 & =s_{1}+8 s_{2} z_{s}+48 s_{3} z_{s}^{2}+8 s_{4} z_{s}^{2} \ldots, \\
1 & =2 v_{1} z_{v}+24 v_{2} z_{v}^{2}+4 v_{3} z_{v}^{2} \ldots
\end{aligned}
$$

The two parameters $z_{s}(\beta)$ and $z_{v}(\beta)$ can be used to optimize the properties of $D$ while at the same time maintaining unchanged the equations (2.12) which are equivalent to the Ginsparg-Wilson equation.

We emphasize, that these two supplementary conditions are just a practical means to effectively reduce the number of necessary terms in the Dirac 
operator. In some sense they serve as a guiding principle to select more local actions in the huge space of possible actions satisfying the Ginsparg-Wilson condition.

We can now restrict ourselves to a much smaller set of terms in the expansion (2.7) of $D$. Once this set is chosen, $z_{s}$ and $z_{v}$ will be determined by optimizing the properties of the spectrum of $D$ near the origin. The parameter $z_{s}$ is fixed by the requirement that the small eigenvalues of $D$ fall on the Ginsparg-Wilson circle (i.e. $m=0$ ); $z_{v}$ can e.g. be fixed by requiring the slope of the $\pi$-dispersion relation to be equal to 1 . In this preliminary study we will simply determine $z_{v}$ by optimizing the alignment of larger eigenvalues along the circle.

It is important to remark, that already including only a few terms in the expansion (2.7) of $D$ and solving the corresponding system (2.12) considerably orders the small eigenvalues of $D$ (see below). Thus no complicated fine-tuning procedure is necessary for $z_{s}$ and $z_{v}$ and they can simply be determined by analyzing the spectrum of $D$ on a few background gauge field configurations.

Finally we remark, that another feature which one would like to implement is $\mathcal{O}(a)$ improvement 19 . It is known, that an exact solution of the Ginsparg-Wilson equation is already $\mathcal{O}(a)$ improved. However, here we will discuss only an approximate solution and it is useful to require $\mathcal{O}(a)$ improvement independently of the Ginsparg-Wilson equation. $\mathcal{O}(a)$ improvement may be achieved by adding to $D$ the so-called clover-leaf term $c_{s w} \frac{1}{2} \sigma \cdot F[20]$. At tree level the coefficient $c_{s w}$ equals the factor in front of the Laplace-type contribution to $D$. For non-perturbative improvement $c_{s w}$ can e.g. be determined using the Schrödinger functional [21]. Since we do not attempt to determine $c_{s w}$ non-perturbatively here we quote the condition at tree level (i.e. $c_{s w}=1$ ). It is obtained by expanding the terms in the tensor sector of our $D$ for small lattice spacing, extracting their $\frac{1}{2} \sigma \cdot F$ content and setting the factor in front of this term equal to the factor of the Laplace-term contribution of $D$. One finds (compare also [16])

$$
s_{2}+12 s_{3}+4 s_{4} \ldots=4 t_{1}+32 t_{2}+16 t_{3} \ldots .
$$

It is straightforward to include $c_{s w}$ as a free parameter in (3.8) but as already remarked above we do not attempt a nonperturbative evaluation of $c_{s w}$ here and thus work with the tree level equation (3.8) throughout this paper.

At this point we would like to comment on the fate of the doubler modes. When analyzing our solutions for the free case we always find that the eigenvalues for the doublers (at least one component $p_{\mu}$ of the four-momentum 
equals $\pi$ ) are located near 2 in the complex plane as is the case for exact, doubler-free solutions of the Ginsparg-Wilson equation. Thus our approximation scheme already takes care of the doublers and we do not need to supplement the boundary condition (3.1) by an additional equation for the doublers.

\subsection{Truncation of $D$ and numerical solution of the system of coupled equations}

In the last section the set of equations (2.12) together with the boundary conditions (3.7) and (3.8) was discussed. In principle the system (2.12) contains infinitely many equations, each of them with infinitely many terms. Also the boundary conditions contain infinitely many terms. The next step thus will be a truncation of the expansion (2.7) for $D$ which will reduce the infinite problem to a finite problem.

Any reasonable truncation should have a parameter which controls the size of the remainder of the approximation. In our case such a parameter is given by the length of the paths in each term in the expansion (2.7) of $D$. We find that roughly the size of the coefficients $s_{i}^{\alpha}, v_{i}^{\alpha}, t_{i}^{\alpha}, a_{i}^{\alpha}, p_{i}^{\alpha}$ decreases exponentially as the length of the paths in the corresponding terms increases (compare Table 3 in the appendix). Thus neglecting terms with longer paths provides a natural cutoff scheme for the expansion of $D$. The exponential decrease of the coefficients not only justifies our cutoff, but is also important for the physics described by $D$. A solution of the Ginsparg-Wilson equation cannot be ultra-local 18. However, in order to remain in the correct universality class, $D$ has to be local, i.e. $D_{x, y}$ has to decrease exponentially as the distance $|x-y|$ on the lattice increases. In Fig. 1 we plot the size of the coefficients as a function of $|x-y|$ on a logarithmic scale. We show the coefficients for the free case (full circles are used to represent all the coefficients) as well as those used for the quenched gauge field ensemble (triangles) from the Wilson gauge action at $\beta=6.0$ (cf. Table 3). The coefficients for the other three ensembles show a similar behavior. The size of the coefficients is consistent with being bound by an exponential decay with $|x-y|$. Thus we have verified, that we approximate a healthy, local solution of (1.1).

Let us now come to the more practical aspects of the truncation. It is clear that adding more terms in the expansion (2.7) of $D$ improves the quality of the approximation of a solution of the Ginsparg-Wilson equation. On the other hand each new term drives up the numerical cost. Here we

present an operator which manages to give a decent approximation of a solution of (1.1) but at the same time is still relatively cheap to simulate. 


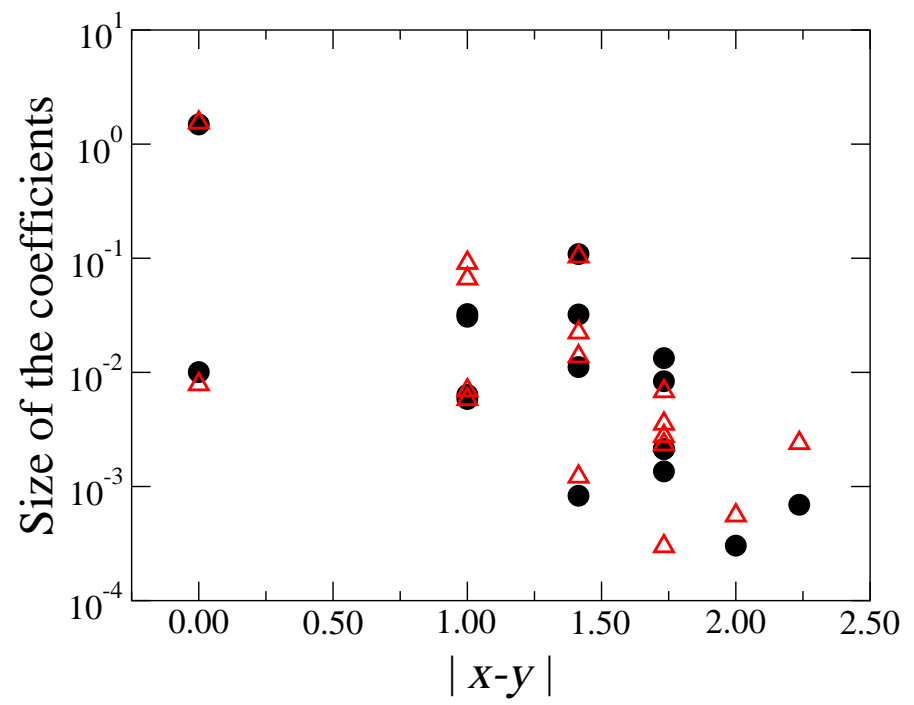

Figure 1: Size of the coefficients as a function of the distance $|x-y|$ on the lattice. We use full circles for all the coefficients in the free case, the triangles represent the coefficients of the quenched ensemble at $\beta=6.0$ (cf. Table 3).

When constructing the operator we started with a simple parametrization and added only terms which gave rise to a considerable improvement of the spectral properties. We stopped adding terms when our $D$ had a satisfactory balance of good chiral properties and low numerical cost.

Also the choice of equations from the set (2.12) allows for some freedom (the boundary conditions (3.7) and (3.8) are always implemented). This freedom again goes back to the non-existence [18] of ultra-local solutions of (1.1). If we could solve all equations (2.12) for a finite parametrization (2.7) of $D$ this would, however, amount to an ultra-local solution. The loophole out of this dilemma is the fact that the system (2.12) is always overdetermined. Thus we can only solve the equations corresponding to the leading terms of the expansion (2.11) of $E$, where again the length of the paths is the expansion parameter.

The solution which we present here has altogether 17 terms in the expansion (2.7) of $D$. The maximum path length is 4 and we only included terms in the scalar, vector and tensor sectors. Except for one term $\left(s_{6}\right)$ all terms have paths on the hypercube. For the vector and tensor sectors we allowed only terms up to length 3 on the hypercube, since longer terms in 
these sectors create many new entries in the fermion matrix which quickly increase the numerical cost. A detailed description of the terms used in our $D$ and the values of the coefficients are given in the appendix in Table 2 and Table 3. Besides the three boundary conditions (3.7), (3.8) we implement 14 equations from the system (2.12) to match the number of expansion coefficients (17). These equations correspond to the shortest terms with paths on the hypercube contributing to the expansion (2.11) of $E$. We remark, that it was straightforward to exactly solve the equations (2.12), (3.7), (3.8) with a standard solver [22] and we also found that the system is very stable, i.e. we found only a single solution for our setting.

\section{Properties of our Dirac-operator}

In this section we analyze the properties of our Dirac operator for different ensembles of background gauge fields. We start with the analysis of the free case, followed by a study using quenched SU(3) configurations generated with the standard Wilson action for the gauge fields. We conclude with analyzing gauge fields from the improved Lüscher-Weisz action.

\subsection{The free case}

For the free case it is possible to completely diagonalize the Dirac operator using Fourier transformation. In Fig. 2 we show the free spectrum of our $D$ in the complex plane. In addition to the eigenvalues (symbols) we also show the Ginsparg-Wilson circle (full curve) which supports the spectrum of any exact solution of (1.1). Throughout this article we present our spectra for $8^{4}$ lattices. This is a relatively small volume for usual standards, however, the Dirac operator studied here is ultralocal and its extent is well within the lattice size.

Let us briefly discuss some features of the free spectrum. It is obvious, that our approach optimizes the alignment of the eigenvalues along the circle for the eigenvalues in the physical branch, i.e. the eigenvalues in the vicinity of the origin. The alignment of the eigenvalues in the doubler branches is less perfect. The important feature, however, is the clear separation of the doubler modes and the physical eigenvalues. We furthermore found, that adding additional terms in (2.7) systematically improves the situation also for the doublers. This property of our approach of first optimizing the physical branch and aligning the doubler modes along the circle when adding higher terms persists also when analyzing the spectrum for nontrivial background gauge fields and was observed also for the 2-d case [15]. 


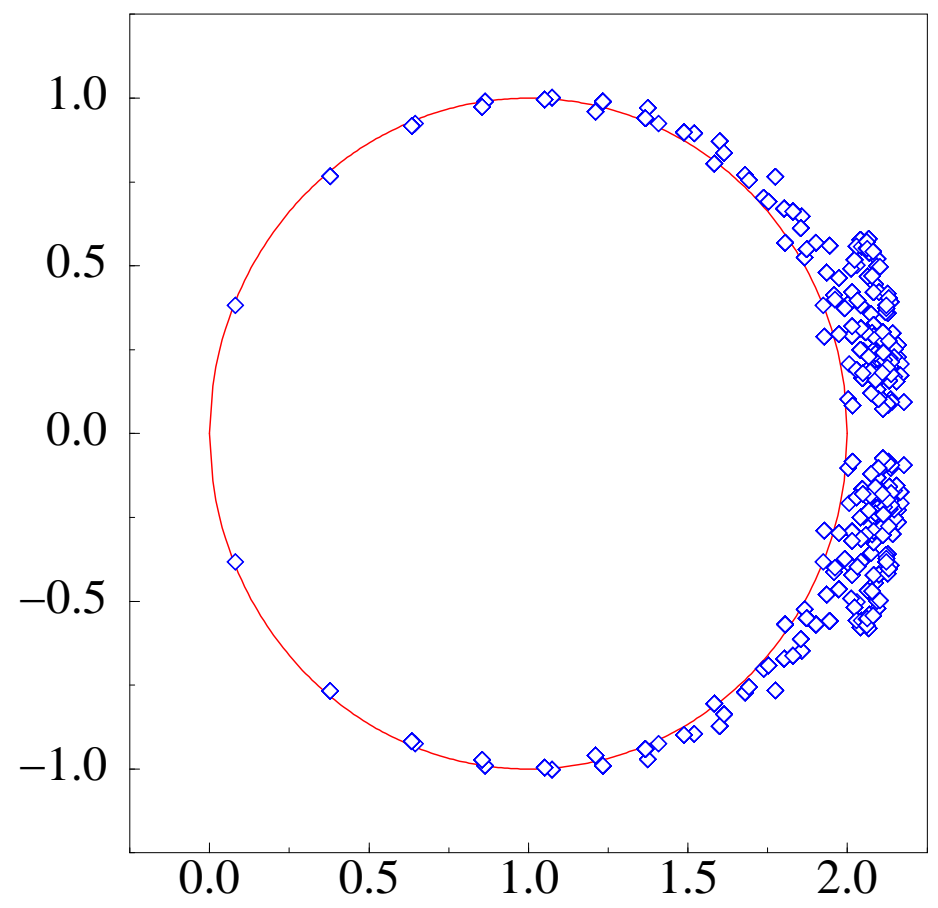

Figure 2: Spectrum of our Dirac operator for the free case in the complex plane. The represent the eigenvalues. We also show the Ginsparg-Wilson circle, i.e. the circle of radius 1 and center 1 .

At this point it is interesting to compare our method to the study of truncated perfect actions for free fermions [17, 23, 24]. For free fermions the perfect action can be computed explicitly 8$]$ as an infinite series and is a solution of the Ginsparg-Wilson equation. After truncation to e.g. only terms on the hypercube the result is an approximate solution of the GinspargWilson equation for the free case.

This truncated perfect action will however differ from our approach in two important aspects: Firstly actions for free fermions only contain scalar and vector terms. Terms in the tensor, pseudovector and pseudoscalar sectors vanish identically when no gauge field is coupled. Thus e.g. terms in the tensor sector which are necessary for $\mathcal{O}(a)$ improvement and also play an important role for a smooth spectrum in the physical branch [25] have to be included a posteriori. Secondly for each possible endpoint of a fermion 
path the truncated perfect action for free fermions provides only a single coefficient. On the other hand, our symmetry analysis (compare (2.7)) shows, that different paths leading to this endpoint can come with different coefficients. Thus the distribution of the single coefficient of the free perfect action among the various coefficients allowed by the exact symmetry analysis is an additional task.

Overcoming these problems, Bietenholz 24] presented interesting results for an approximate solution of the Ginsparg-Wilson equation in 4-d. After introducing fat links and a link amplification factor for the coefficients of the paths, an approximate solution of the Ginsparg-Wilson equation also for the case with gauge fields is obtained from the truncated perfect action. It is interesting to note that the spectra presented in [24] show a behavior quite contrary to the spectra for our $D$ : They come with a very smooth doubler branch while the modes in the physical branch have quite large fluctuations. Bietenholz expresses confidence that the physical branch can be smoothened by adding tensor terms but expects that these terms will at the same time destroy the good alignment of the eigenvalues in the doubler branch [24].

\subsection{Results for quenched gauge configurations generated with the Wilson gauge action}

After having studied the properties of our $D$ for the free case we are now analyzing the Dirac operator in ensembles of quenched SU(3) gauge field configurations. We concentrate on the branch of the spectrum near small eigenvalues, which is the one most relevant in the continuum limit. We compute the eigenvalues in this physical branch of the spectrum and compare them with the eigenvalues for the Wilson operator (3.4). The values of $\kappa$ used for the Wilson operator (3.4) can be found in Table 11 and will be discussed below.

For the case of non-trivial background gauge fields the Dirac operator can no longer be diagonalized completely unless one uses very small lattices. Here we use the Implicitly Restarted Arnoldi Method [26] to compute eigenvalues in the physical branch of the spectrum, i.e. near the origin. The method allows to specify a search criterion for the eigenvalues and we use this feature to compute the eigenvalues with the smallest real parts. In order to get insight into the typical behavior of the spectrum of our $D$, we analyzed the eigenvalues on 4 ensembles of quenched gauge fields. Each ensemble consists of 20 well decorrelated configurations. Two of the ensembles were generated with the standard Wilson action at $\beta=6.0$ and $\beta=5.85$. They will be discussed in this subsection. The other two ensembles were generated 

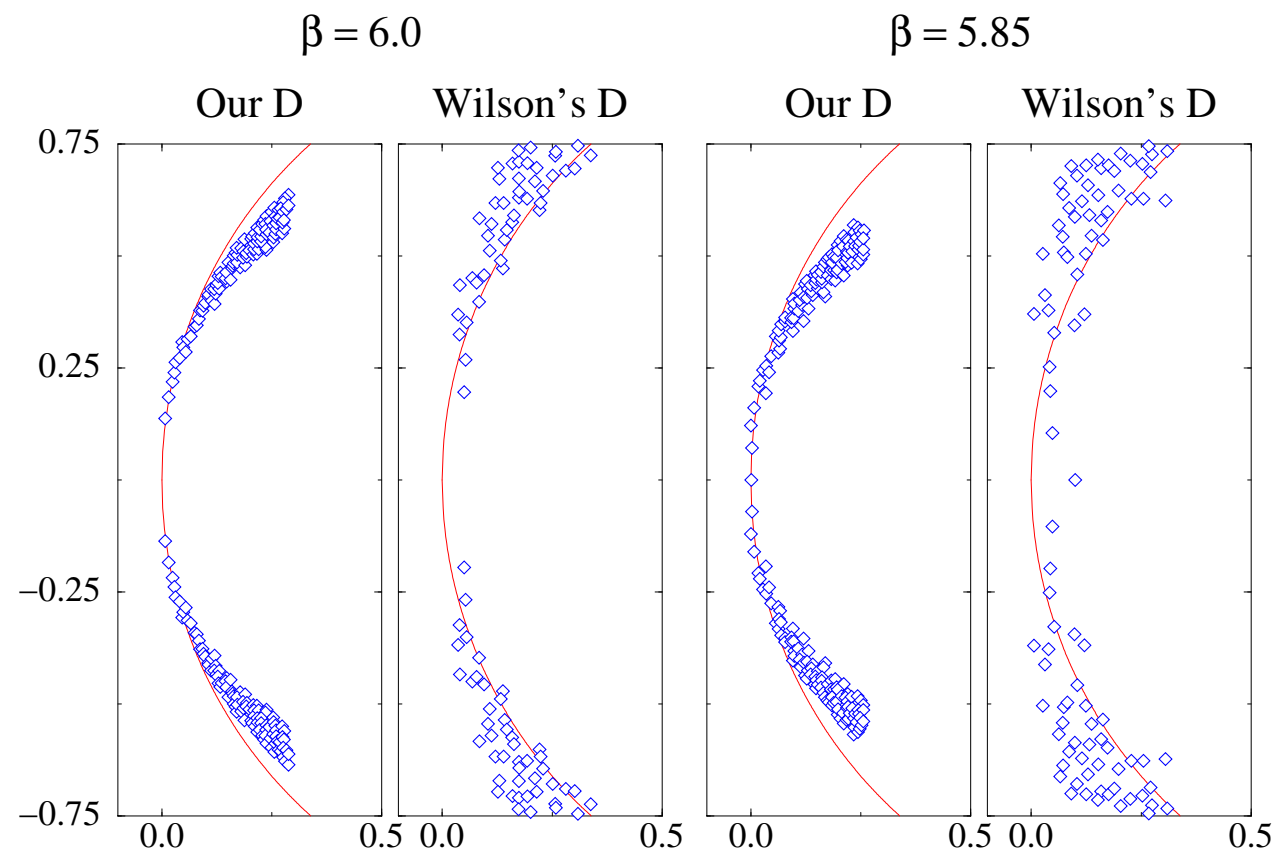

Figure 3: The physical branch of spectra of Dirac operators in the complex plane for quenched $\mathrm{SU}(3)$ gauge field configurations generated using the Wilson gauge action. From left to right we show: The spectrum of our $D$ at $\beta=6.0$, for Wilson's Dirac operator at $\beta=6.0$, our $D$ at $\beta=5.85$ and finally for Wilson's $D$ at $\beta=5.85$.

using the improved Lüscher-Weisz action [27] and will be analyzed in the next subsection. For each gauge field configuration we computed 60 eigenvalues in the physical branch for both our Dirac operator as well as for the Wilson-Dirac operator. For the plots in Fig. 3 and Fig. 5 we increased the number of computed eigenvalues to 200. All these calculations were done on $8^{4}$ lattices with anti-periodic boundary conditions for the Dirac operator.

In Fig. 3 we show the physical branch of the spectrum of our operator for quenched gauge field configurations generated with the standard Wilson gauge action at $\beta=6.0$ and $\beta=5.85$. We compare these spectra with the spectra of the Wilson-Dirac operator on the same configurations. The symbols are the numerically computed eigenvalues and the full curve is the Ginsparg-Wilson circle.

It is obvious, that for our $D$ the eigenvalues are more ordered than for 
Wilson's $D$ for both $\beta=6.0$ and $\beta=5.85$. The spread of the eigenvalues of our $D$ is reduced when compared to the Wilson case and the eigenvalues tend to order near a curve. However, what is also obvious is the fact that although this curve touches the origin, it also bends away from the GinspargWilson circle as the size of the imaginary parts increases. As can be seen from the Fourier transform in the free case, it is essentially the vector terms in the expansion (2.7) contributing to the imaginary parts of the eigenvalues. As we have already remarked above, we allowed only for a relatively small number of vector terms (compare Table 2) in order to reduce the numerical cost. We thus attribute the bending away of the eigenvalues to this reduced approximation of the vector sector. In the next section we will, however, demonstrate, that our parametrization of the vector sector is sufficient if one uses improved gauge field actions.

It is also interesting to study the behavior of the real eigenmodes - the $\beta=5.85$ part of Fig. 3 shows one real eigenvalue. Typically the fluctuations of the real eigenvalues of the Wilson-Dirac operator are relatively large. In particular for so-called exceptional configurations (in the sense of a breakdown of the matrix inversion) a real mode has fluctuated so heavily towards small values that it compensates for the bare quark mass and the resulting zero mode leads to a breakdown of the matrix inversion. We found, and Fig. 3 shows an example, that our $D$ strongly suppresses the fluctuations of the real modes and turns them into almost perfect zero modes. We thus expect that at nonzero bare quark mass our $D$ considerably reduces the numerical difficulties with exceptional configurations.

Let us now discuss the overall fluctuation of the rest of the physical branch of the spectrum. An exact solution of the Ginsparg-Wilson equation has its spectrum on the Ginsparg-Wilson circle and only the density of eigenvalues on this circle will fluctuate. The eigenvalues of the WilsonDirac operator, on the other hand, have relatively large fluctuations in all directions. When analyzing the ensemble of 20 configurations for both $\beta=$ 6.0 and $\beta=5.85$ we found that for our $D$ the fluctuations of the physical edge of the spectrum are considerably suppressed when compared to the Wilson-Dirac operator. In order to quantify this statement we fit a circle of the form

$$
y= \pm i \sqrt{1-(1+\varepsilon-x)^{2}},
$$

to the 10 smallest eigenvalues in the physical branch omitting the exactly real eigenvalues, because they deviate significantly in the case of the Wilson operator (compare above). Eq. (4.1) describes a circle of radius 1 around $(1+\varepsilon, 0)$ in the complex plane. The parameter $\varepsilon$ of the fit gives the position 
where the circle crosses the real axis. The fluctuation of $\varepsilon$ provides a measure for the fluctuation of the eigenvalues in the physical branch of the spectrum.
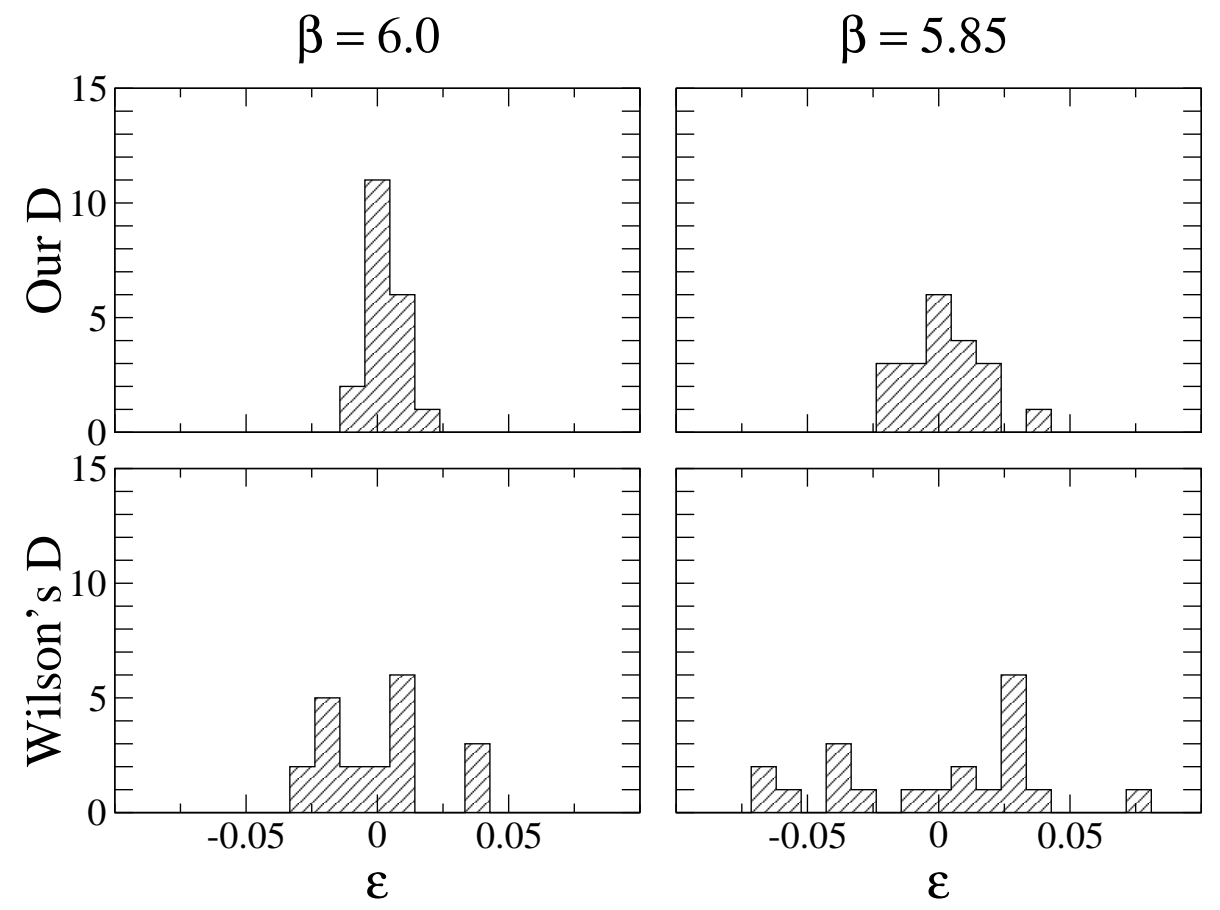

Figure 4: Histograms indicating the fluctuation of $\varepsilon$ (i.e. the fluctuation of the physical branch of the spectrum) for two ensembles of 20 quenched gauge field configurations generated with the Wilson gauge action at $\beta=6.0$ (left column) and $\beta=5.85$ (right column). The top row shows the results for our $D$, the bottom row displays the results for the Wilson-Dirac operator.

In Fig. 1 we show for each of our ensembles a histogram of the values of $\varepsilon$ for all 20 configurations, comparing the results for our Dirac operator with those for Wilson's $D$. It can be seen, that our $D$ has considerably reduced the fluctuations of the physical edge of the spectrum, in particular for $\beta=6.0$.

At this point we can also comment on the determination of the critical $\kappa$ for the Wilson-Dirac operator (3.4). Since the first term is proportional to the unit matrix, it is sufficient to diagonalize the hopping matrix $H$. The value of $\kappa$ was then adjusted such that the distribution of $\varepsilon$ is centered at 0 . The values of $\kappa$ obtained for our 4 ensembles are given in Table 1 below. 


\begin{tabular}{cccccc}
\hline & free case & \multicolumn{2}{c}{ Wilson action } & \multicolumn{2}{c}{ Lüscher-Weisz action } \\
& & $\beta=6.0$ & $\beta=5.85$ & $\beta_{1}=8.45$ & $\beta_{1}=8.15$ \\
\hline$\frac{1}{3}<U_{p l}>$ & 1.0 & $0.594(1)$ & $0.576(1)$ & $0.652(1)$ & $0.633(1)$ \\
$\kappa$ & 0.5 & 0.624 & 0.642 & 0.607 & 0.623 \\
\hline
\end{tabular}

Table 1: Parameters for our ensembles of quenched gauge field configurations and the free case. We list the expectation value $\left\langle U_{p l}>/ 3\right.$ of the plaquette and the critical $\kappa$ we use for the Wilson operator.

\subsection{The effect of improving the gauge action}

In this section we discuss the effect of improving the gauge field on the spectral properties of the Dirac operator. In the context of domain wall fermions it has been found that improving the gauge field action allows to work with a smaller extension of the 5-th direction [28]. Also for the 4 -d setting it is worth testing if improving the gauge field action leads to an improvement of the spectral properties of $D$ in particular since from a numerical point of view improving the gauge action is an inexpensive measure.

In order to analyze the effects of gauge improvement we generated 2 ensembles of 20 gauge configurations each, using the Lüscher-Weisz action [27, 29]. The parameters were adjusted such that the physical scale (the effective string tension) approximates the two scales of our ensembles from the standard Wilson gauge action at $\beta=6.0$ and $\beta=5.85$. Our estimator for the effective string tension is based on Wilson loops up to extent 3 only and therefore not to be compared with the values derived on larger lattices with a detailed finite size analysis. However, our principal incentive was not to obtain such a precise value, but to identify the values of the gauge couplings where the two gauge actions have roughly the same scale.

To be specific, we use the setting for the improved gauge action as presented in 29] but with only the rectangle term. Explicitly, the gauge field action reads

$$
S[U]=\beta_{1} \sum_{p l} \frac{1}{3} \operatorname{Re} \operatorname{Tr}\left(1-U_{p l}\right)+\beta_{2} \sum_{r t} \frac{1}{3} \operatorname{Re} \operatorname{Tr}\left(1-U_{r t}\right),
$$

where the first sum is over all plaquettes and the second sum over all $2 \times 1$ rectangles. $\beta_{1}$ is the driving parameter while $\beta_{2}$ can be computed from $\beta_{1}$ 
using tadpole improved perturbation theory [30] giving [29]

$$
\beta_{2}=-\frac{\beta_{1}}{20 u_{0}^{2}}\left[1+0.4805 \alpha_{s}\right] .
$$

with

$$
u_{0}=\left(\frac{1}{3} \operatorname{Re} \operatorname{Tr}\left\langle U_{p l}\right\rangle\right)^{1 / 4}, \alpha_{s}=-\frac{\ln \left(\frac{1}{3} \operatorname{Re} \operatorname{Tr}\left\langle U_{p l}\right\rangle\right)}{3.06839} .
$$

The coupling $\beta_{1}$ is determined self-consistently with $u_{0}$ and $\alpha_{s}$ for a given $\beta_{1}$. We adjust the parameter $\beta_{1}$ such that the physical scale approximates the two scales of our ensembles from the standard Wilson gauge action at $\beta=6.0$ and $\beta=5.85$, i.e. such that the effective string tension of the improved ensemble (as discussed above) roughly matches the value of the ensemble from the standard Wilson action. The resulting values are $\beta_{1}=8.45$ which corresponds to $\beta=6.0$ for the Wilson action and $\beta_{1}=8.15$ corresponding to $\beta=5.85$. We list our results for the plaquette expectation value and the values of the critical $\kappa$ determined for the Wilson-Dirac operator in Table 1 .

Like for the ensembles generated with the Wilson gauge action also here we computed for each configuration 60 eigenvalues in the physical branch for both, our $D$ as well as for Wilson's Dirac operator. When inspecting these eigenvalues, one finds that the spectral properties of our $D$ are considerably improved. In Fig. 5 we show examples of spectra for one configuration from each of the ensembles. As in the last section we show 200 eigenvalues in the physical branch for our $D$ as well as for the Wilson-Dirac operator both for the same gauge configuration.

When comparing these plots to Fig. 3 one finds that the eigenvalues of our $D$ are somewhat better aligned along a single curve, i.e. the distribution is narrower. Also the imaginary parts are larger and the curve is following the Ginsparg-Wilson circle much closer than it was the case for the standard Wilson gauge action. The improved spectral properties of $D$ can be attributed to the following feature of the improved gauge field ensemble: When comparing the plaquette expectation value of the improved ensemble with that of the Wilson ensemble at the same physical scale one finds that improvement brings the plaquettes considerably closer to 1 (see Table 1). A second, although smaller effect, might be the better rotational properties of the gauge fields from the improved action 29.

We remark, that also the eigenvalues of the Wilson-Dirac operator are slightly more ordered when using the improved gauge field action. It is expected that this already could help to improve on the problems with the singularities in the inverse square root of the overlap operator [31. 

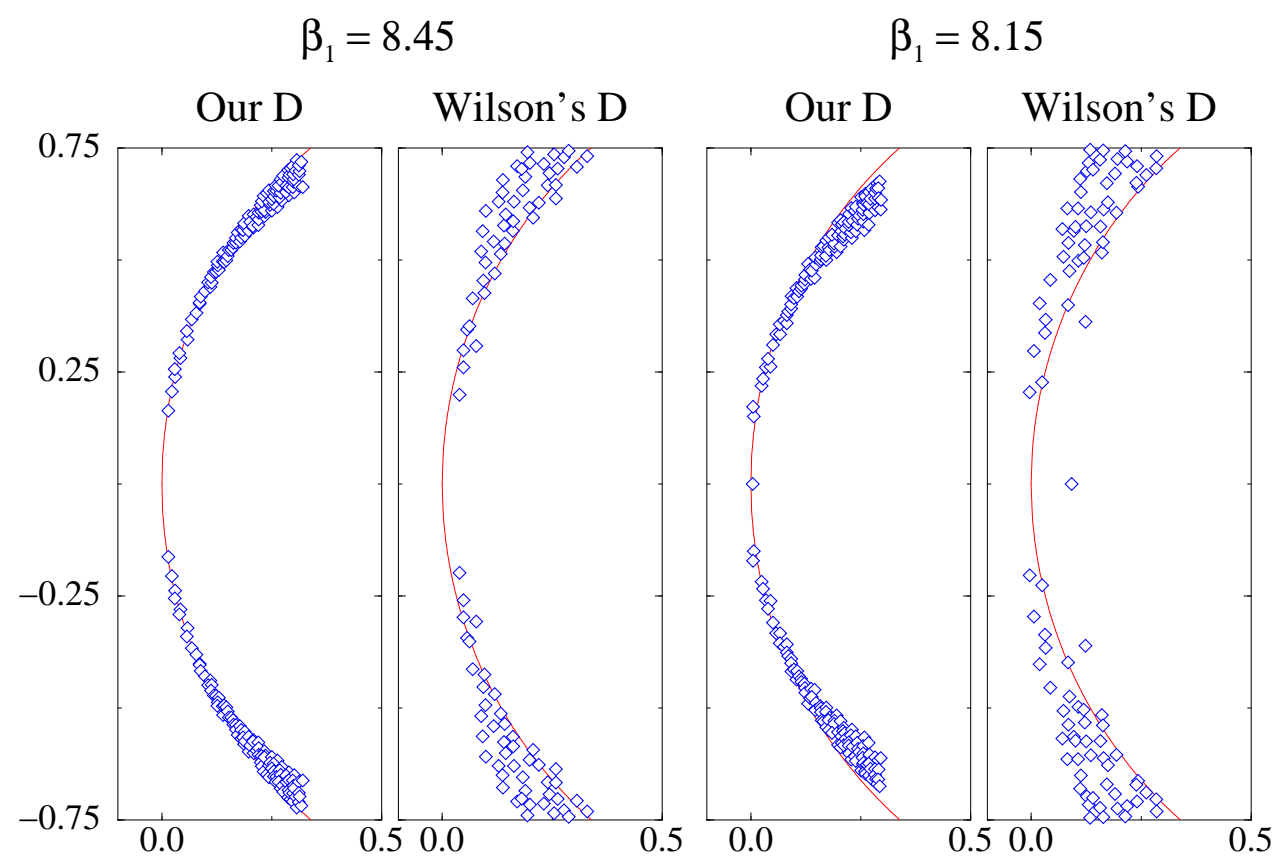

Figure 5: The physical branch of spectra of Dirac operators in the complex plane for quenched $\mathrm{SU}(3)$ gauge field configurations generated using the Lüscher-Weisz gauge action. From left to right we show: The spectrum of our $D$ at $\beta_{1}=8.45$, for Wilson's Dirac operator at $\beta_{1}=8.45$, our $D$ at $\beta_{1}=8.15$ and finally again Wilson's $D$ at $\beta_{1}=8.15$.

We conclude this subsection with repeating last section's analysis of the fluctuations of the physical branch, now for the improved ensemble. When comparing Fig. 6 and Fig. 1 one finds, that for both our $D$ as well as for the Wilson-Dirac operator the distribution of $\varepsilon$ becomes slightly narrower when using the improved gauge action.

We thus find that using the improved gauge action is a numerically inexpensive measure leading to a considerable improvement of the spectral properties of the lattice Dirac operator. In particular we observe that improving the gauge fields allows to work with a relatively small number of terms in the vector sector of $D$ and still obtain a good approximation of a solution of the Ginsparg-Wilson equation. 

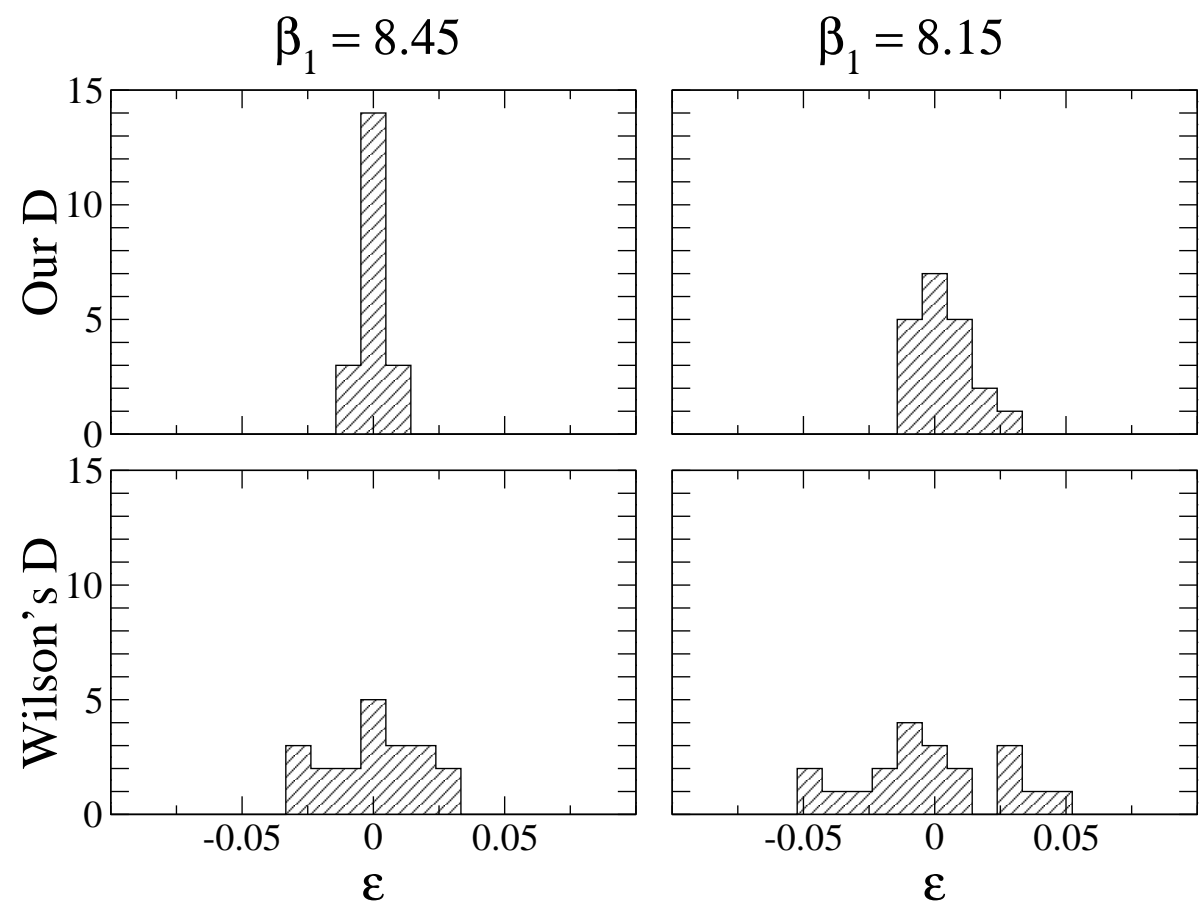

Figure 6: Fluctuation of the physical branch of the spectrum for the two ensembles from the Lüscher-Weisz action at $\beta_{1}=8.45$ (left column) and $\beta_{1}=8.15$ (right column). The top row shows the results for our $D$, the bottom row displays the results for the Wilson-Dirac operator.

\section{Discussion}

In this article we have presented first tests in 4-d for a new method of constructing approximate solutions of the Ginsparg-Wilson equation. The most general Dirac operator on the lattice is systematically expanded in a series of simple operators. The Ginsparg-Wilson equation turns into a set of coupled quadratic equations for the expansion coefficients. For a finite parametrization of $D$ these equations can be solved and the corresponding $D$ is an approximate solution of the Ginsparg-Wilson equation. We implement boundary conditions which allow to work with a very economical parametrization of $D$ and still obtain a good approximation of a solution of the Ginsparg-Wilson equation. Our framework allows to systematically include $\mathcal{O}(a)$ improvement.

We find that our method has the intriguing feature that already a few 
terms lead to a good alignment on the Ginsparg-Wilson circle for the physical modes. The alignment of the doubler branch can be systematically improved by adding additional terms in the parametrization of $D$. We have demonstrated that the fluctuations of the eigenvalues in the physical branch are much smaller than e.g. for the Wilson-Dirac operator and we expect that our $D$ gets essentially rid of the problems with exceptional configurations. Finally we have observed that using an improved action for the gauge fields considerably improves the spectral properties of our $D$.

At this point we would like to comment on the numerical cost of our Dirac operator. So far we have not implemented an optimized matrix-vector multiplication for our $D$. Our test program was kept general in order to be able to test Dirac operators with more terms than the ones we present here. Thus we give a theoretical estimate of the numerical cost of our Dirac operator and compare it to the cost of the standard Wilson-Dirac operator. To leading order the numerical cost is determined by the number of nonvanishing entries in the fermion matrix. For our $D$ this number is given by 297 where we have factored out the volume and a factor of 12 for color and spinor structure. The corresponding number for Wilson's Dirac operator is 17. Thus to leading order we find a factor of $297 / 17=17.47$ when comparing the numerical cost for our $D$ with Wilson's Dirac operator. This estimate, however, assumes that the all necessary products of the link variables can be stored. This is possible for smaller lattices up to $10^{4}$, but for larger lattices only pieces of paths can be stored. With a good storage strategy the additional cost for building up the longer paths can be kept below a factor of 2 . We estimate our $D$ to be approximately 25 times more expensive when compared to Wilson's $D$. Finally, we also found that the Arnoldi diagonalization routine we use typically needs $35 \%$ less matrix multiplications for our $D$ as compared to Wilson's Dirac operator.

Let us now come back to the three possible applications of our $D$ which we mentioned in the introduction:

- We think that it would be interesting to use our $D$ for analyzing the pion spectrum in the quenched approximation. The fact that the fluctuations of the spectrum near the origin are highly suppressed should allow one to perform simulations at small bare quark masses without running into problems with exceptional configurations. Also studies of the distribution of the eigenvalues of our $D$ could be used to compute the chiral condensate from the Banks-Casher formula.

- Our successful construction of an approximate solution for the GinspargWilson equation also seems to be good news for the project of con- 
structing perfect fermion actions using block spin transformations. It seems quite feasible to construct a sufficiently rich parametrization with only 15 to 20 parameters. It seems to us that in the scalar sector it is necessary to include relatively many terms, while in the higher sectors one can try to be more economical, in particular when using improved or perfect gauge actions.

- Finally our $D$ is also a good candidate for an improved starting operator $D_{0}$ in the overlap projection (1.2): It is already much closer to a solution of the Ginsparg-Wilson equation than e.g. the standard Wilson-Dirac operator which is usually used for $D_{0}$. Thus we expect that an expansion for the inverse square root converges faster when using our $D$ as starting operator.

Acknowledgements: The authors would like to thank Mark Alford, Wolfgang Bietenholz, Richard Brower, Peter Hasenfratz, Keh-Fei Liu, Ferenc Niedermayer, Jack Verbaarschot and Uwe-Jens Wiese for interesting discussions. We also thank Mark Alford and Peter Lepage for sharing their computer program for generating the improved gauge field ensembles. The numerical computations were done on the NICse Alpha Linux cluster at NIC Jülich. Christof Gattringer thanks the INT at the University of Washington in Seattle, where part of this work was done, for its kind hospitality. 


\section{A Technical appendix}

In this appendix we describe in more detail the terms in our Dirac operator and give the values for the coefficients which we use for the 4 ensembles of quenched gauge field configurations.

As has been pointed out in Section 2.1, the most general Dirac operator $D$ can be expanded in the series (2.7). Each term in this series is characterized by three pieces:

1. a generator of the Clifford algebra,

2. a group of paths,

3. a real coefficient.

The paths within a group can have different signs which are determined by the symmetries, C, P, $\gamma_{5}$-hermiticity and rotation invariance (for the operation of these symmetries see Section 2.1). The symmetries also determine which paths are grouped together. Thus it is sufficient to characterize a group of paths by a single generating path and all the other paths in the group as well as their relative their sign factors can be determined by applying the symmetries.

In addition, for the vector and tensor terms appearing in our $D$ it is sufficient to give the paths only for one vector (tensor) since rotation invariance immediately fixes the structure for the other vector (tensor) terms. In order to describe our $D$ we start with listing the three determining pieces for each term in Table 2 .

In Section 4 we analyze our $D$ in different ensembles of quenched background gauge fields. In particular we use ensembles generated with the standard Wilson action at $\beta=6.0$ and $\beta=5.85$ and ensembles from the Lüscher-Weisz action at $\beta_{1}=8.45$ and $\beta_{1}=8.15$ (for more details see Section 4.3). For all these configurations we use the same parametrization of $D$. Only the values of the coefficients $s_{i}^{\alpha}, v_{i}^{\alpha}$ and $t_{i}^{\alpha}$ and the two normalization factors $z_{s}$ and $z_{v}$ for the boundary conditions differ. In Table 3 we list their values. 


\begin{tabular}{ccc}
\hline & & \\
Clifford generator & Generating path & Name of coefficient \\
\hline $\mathbb{I}$ & $<>$ & $s_{1}$ \\
$\mathbb{I}$ & $<1>$ & $s_{2}$ \\
$\mathbb{I}$ & $<1,2>$ & $s_{3}$ \\
$\mathbb{I}$ & $<1,2,3>$ & $s_{5}$ \\
$\mathbb{I}$ & $<1,1,2>$ & $s_{6}$ \\
$\mathbb{I}$ & $<1,2,3,4>$ & $s_{8}$ \\
$\mathbb{I}$ & $<1,2,-1,3>$ & $s_{10}$ \\
$\mathbb{I}$ & $<1,2,-1,-2>$ & $s_{11}$ \\
$\mathbb{I}$ & $<1>$ & $s_{13}$ \\
\hline$\gamma_{1}$ & $<1,2>$ & $v_{1}$ \\
$\gamma_{1}$ & $<1,2,3>$ & $v_{2}$ \\
$\gamma_{1}$ & $<2,1,3>$ & $v_{4}$ \\
$\gamma_{1}$ & $<1,2>$ & $v_{5}$ \\
\hline$\gamma_{1} \gamma_{2}$ & $<1,2,3>$ & $t_{1}$ \\
$\gamma_{1} \gamma_{2}$ & $<1,3,2>$ & $t_{2}$ \\
$\gamma_{1} \gamma_{2}$ & $<1,2,-1>$ & $t_{3}$ \\
$\gamma_{1} \gamma_{2}$ & $t_{5}$ \\
\hline
\end{tabular}

Table 2: Description of the terms in our $D$. 


\begin{tabular}{cccccc}
\hline & free case & \multicolumn{2}{c}{ Wilson action } & \multicolumn{2}{c}{ Lüscher-Weisz action } \\
& & $\beta=6.0$ & $\beta=5.85$ & $\beta_{1}=8.45$ & $\beta_{1}=8.15$ \\
\hline$s_{1}$ & +1.488513 & +1.541745 & +1.536335 & +1.545142 & +1.546343 \\
$s_{2}$ & -0.030753 & -0.066240 & -0.069085 & -0.061723 & -0.063831 \\
$s_{3}$ & -0.011132 & -0.013808 & -0.014036 & -0.014045 & -0.013612 \\
$s_{5}$ & -0.002128 & -0.002763 & -0.002844 & -0.002571 & -0.002690 \\
$s_{6}$ & -0.000691 & +0.002396 & +0.002649 & +0.002228 & +0.002198 \\
$s_{8}$ & -0.005842 & -0.005813 & -0.005786 & -0.005415 & -0.005811 \\
$s_{10}$ & -0.000303 & -0.000557 & -0.000593 & -0.000512 & -0.000526 \\
$s_{11}$ & -0.000830 & -0.001217 & -0.001241 & -0.001181 & -0.001200 \\
$s_{13}$ & -0.010061 & +0.007853 & +0.007918 & +0.007831 & +0.007788 \\
\hline$v_{1}$ & +0.032416 & +0.091060 & +0.096935 & +0.107048 & +0.087543 \\
$v_{2}$ & +0.032158 & +0.022333 & +0.022054 & +0.017838 & +0.022207 \\
$v_{4}$ & +0.008365 & +0.006828 & +0.006007 & +0.007926 & +0.007540 \\
$v_{5}$ & -0.013326 & +0.000299 & +0.000423 & +0.001779 & -0.000364 \\
\hline$t_{1}$ & -0.108727 & -0.103545 & -0.103318 & -0.102314 & -0.103576 \\
$t_{2}$ & -0.002129 & -0.003544 & -0.003715 & -0.003206 & -0.003394 \\
$t_{3}$ & +0.001356 & +0.002333 & +0.002444 & -0.002198 & +0.002239 \\
$t_{5}$ & -0.006355 & -0.006945 & -0.007266 & -0.006170 & -0.006645 \\
\hline$z_{s}$ & 1.0 & 0.876 & 0.866 & 0.895 & 0.885 \\
$z_{v}$ & 1.0 & 0.868 & 0.885 & 0.846 & 0.854 \\
\hline
\end{tabular}

Table 3: The numerical values of the coefficients $s_{i}^{\alpha}, v_{i}^{\alpha}$ and $t_{i}^{\alpha}$ and the factors $z_{s}$ and $z_{v}$ for the different ensembles of gauge fields. 


\section{References}

[1] P.H. Ginsparg and K.G. Wilson, Phys. Rev. D 25 (1982) 2649.

[2] F. Niedermayer, Nucl. Phys. B (Proc. Suppl.) 73 (1999) 105.

[3] M. Lüscher, Nucl. Phys. B (Proc. Suppl.) 83-84 (2000) 34; H. Neuberger, Nucl. Phys. B (Proc. Suppl.) 83-84 (2000) 67.

[4] M. Creutz, hep-lat/0007032.

[5] H. Neuberger, Phys. Lett. B 417 (1998) 141, Phys. Lett. B 427 (1998) 353.

[6] R. Narayanan and H. Neuberger, Phys. Lett. B 302 (1993) 62, Nucl. Phys. B 443 (1995) 305.

[7] P. Hernandez, K. Jansen and M. Lüscher, Nucl. Phys. B 552 (1999) 363.

[8] W. Bietenholz and U.-J. Wiese, Nucl. Phys. B 464 (1996) 319.

[9] P. Hasenfratz, Nucl. Phys. B (Proc. Suppl.) 63 (1998) 53; P. Hasenfratz, Nucl. Phys. B 525 (1998) 401; P. Hasenfratz, V. Laliena and F. Niedermayer, Phys. Lett. B 427 (1998) 353.

[10] P. Hasenfratz, The Theoretical Background and Properties of Perfect Actions, in: Non-Perturbative Quantum Field Physics, eds. M. Asorey and A. Dobado (World Scientific, 1998).

[11] C.B. Lang and T.K. Pany, Nucl. Phys. B 513 (1998) 645; F. Farchioni, C.B. Lang and M. Wohlgenannt, Phys. Lett. B 433 (1998) 377, F. Farchioni, I. Hip, C.B. Lang and M. Wohlgenannt, Nucl. Phys. B 549 (1999) 364.

[12] F. Farchioni and V. Laliena, Nucl. Phys. B 521 (1998) 337, Phys. Rev. D 58 (1998) 054501.

[13] F. Farchioni, I. Hip and C.B. Lang, Phys. Lett. B 443 (1998) 214.

[14] C. Gattringer, Phys. Rev. D (in print), hep-lat/0003005.

[15] C. Gattringer and I. Hip, Phys. Lett. B 480 (2000) 112.

[16] P. Hasenfratz, S. Hauswirth, K. Holland, Th. Jörg, F. Niedermayer and U. Wenger, hep-lat/0003013. 
[17] W. Bietenholz, Eur. Phys. J. C 6 (1999) 537; W. Bietenholz and I. Hip, Nucl. Phys. B 570 (2000) 423, Nucl. Phys. B (Proc. Suppl.) 83-84 (2000) 600.

[18] I. Horvath, Phys. Rev. Lett. 81 (1998) 4063, Phys. Rev. D 60 (1999) 034510; W. Bietenholz, hep-lat/9901005; I. Horvath, C.T. Balwe and R. Mendris, hep-lat/0006027.

[19] K. Symanzik, in Lecture Notes in Physics, Vol. 153, eds. R. Schrader et al. (Springer, New York, 1982); Nucl. Phys. B 226 (1983) 187, 205.

[20] B. Sheikholeslami and B. Wohlert, Nucl. Phys. B 259 (1985) 572.

[21] M. Lüscher, S. Sint, R. Sommer and P. Weisz, Nucl. Phys. B 478 (1996) 365; K. Jansen et al., Phys. Lett. B 372 (1996) 275.

[22] W.H. Press, S.A. Teutolsky, W.T. Vetterling and B.P. Flannery, Numerical Recipes, Cambridge University Press, Cambridge, 1992.

[23] W. Bietenholz, R. Brower, S. Chandrasekharan and U.-J. Wiese, Nucl. Phys. B (Proc. Suppl.) 53 (1997) 921.

[24] W. Bietenholz, hep-lat/0007017.

[25] C. Gattringer and I. Hip, Nucl. Phys. B 541 (1999) 305.

[26] D.C. Sorensen, SIAM J. Matrix Anal. Appl. 13 (1992) 357, R. B. Lehoucq, D.C. Sorensen and C. Yang, ARPACK User's Guide, SIAM, New York, 1998.

[27] M. Lüscher and P. Weisz, Commun. Math. Phys. 97 (1985) 59; Erratum: 98 (1985) 433; G. Curci, P. Menotti and G. Paffuti, Phys. Lett. 130 B (1983) 205, Erratum: 135 B (1984) 516.

[28] CP-PACS Collaboration, A.A. Khan et al, hep-lat/0007014; C. Jung, V. Gadiyak, X. Ji and R.G. Edwards, hep-lat/0007033.

[29] M. Alford, W. Dimm, G.P. Lepage, G. Hockney and P.B. Mackenzie, Phys. Lett. B 361 (1995) 87.

[30] G.P. Lepage and P.B. Mackenzie, Phys. Rev. D 48 (1993) 2250.

[31] K.F. Liu, seminar given at Jefferson Lab, June 2000. 